\title{
Crecimiento y ramificación de Nothofagus alpina y Nothofagus OBLIQUA (NOTHOFAGACEAE) BAJO DIFERENTES CONDICIONES LUMÍNICAS
}

\author{
JAVIER PUNTIERI ${ }^{1,2,}$, CRISTIAN TORRES ${ }^{1}$ y SOLEDAD GHIRARDI ${ }^{1}$
}

\begin{abstract}
Summary: Growth and branching in Nothofagus alpina and Nothofagus obliqua (Nothofagaceae) under different light conditions. Architectural traits of entire main branches and their growth units (GUs) were assessed for trees of Nothofagus alpina and $N$. obliqua developed at three sites with different levels of natural shading (low: roadside, intermediate: nursery, and high: understory). The hierarchical structure of main branches was evaluated by means of: (1) the ratio between the diameters of the secondary branches and that of the parent main branch, and (2) the angles formed between secondary branches and main branches. Understory trees had GUs with less leaves and a thinner and shorter stem compared to those of trees at the less shaded sites. These differences were less notable for more recently-extended GUs. Branching systems became less hierarchical as they aged, but were not significantly different in their hierarchical structure among sites. Main branches of understory $N$. alpina and $N$. obliqua trees may develop for years by producing small and scarcely branched GUs. Main branches of $N$. obliqua trees tend to develop less hierarchical branching systems and to be more negatively affected by shading than those of $N$. alpina.
\end{abstract}

Key words: Architecture, branching pattern, hierarchies, growth unit, morphogenetic gradient, Nothofagus alpina, Nothofagus obliqua, shade tolerance.

\begin{abstract}
Resumen: Se compararon las características arquitecturales de ramas principales completas y de sus unidades de crecimiento (UC) en árboles de Nothofagus alpina y $N$. obliqua desarrollados en tres sitios con diferente nivel de sombreo natural (bajo: banquina, intermedio: vivero, y alto: sotobosque). Se evaluó la estructura jerárquica de las ramas principales mediante: (1) la relación entre el diámetro de las ramas secundarias y el de la rama principal portadora y (2) los ángulos formados entre ramas secundarias y ramas principales. Las ramas principales de los árboles del sotobosque presentaron UC más delgadas y cortas y con menos hojas y ramas que aquellas de los árboles menos sombreados. Estas diferencias disminuyeron para UC extendidas más recientemente. Con el incremento de la edad de los sistemas de ramificación, se observó una disminución en el desarrollo jerárquico de los mismos, pero no se evidenciaron variaciones notables en su jerarquía entre sitios. En ambas especies, las ramas principales de los árboles de sotobosque pueden desarrollarse por años produciendo UC cortas y poco ramificadas. Las ramas principales de $N$. obliqua tienden a ramificarse en forma menos jerárquica y a perder vigor en forma más notable al desarrollarse en la sombra que las de $N$. alpina.
\end{abstract}

Palabras clave: Arquitectura, gradiente morfogenético, jerarquías, patrón de ramificación, Nothofagus alpina, Nothofagus obliqua, tolerancia a la sombra, unidad de crecimiento.

\section{INTRODUCCIÓN}

La arquitectura de las plantas como disciplina se centra en el estudio de los atributos de crecimiento y ramificación de las plantas que se encuentran

${ }^{1}$ INIBIOMA, Universidad Nacional del Comahue - Consejo Nacional de Investigaciones Científicas y Técnicas, Quintral 1250, 8400 Bariloche, Argentina.

${ }^{2}$ Universidad Nacional de Río Negro

*Autor para correspondencia: jgpuntieri@gmail.com genéticamente determinados y constituye un marco de referencia en comparaciones intra- e interespecíficas de la forma de las plantas (Hallé et al., 1978; Tomlinson, 1987). Las variantes arquitecturales de las especies actuales serían el resultado de diferentes respuestas a presiones de selección (Canham, 1988; Cao, 2001), de modo que la probabilidad de que una especie habite un sitio determinado estaría condicionada por sus atributos arquitecturales intrínsecos $\mathrm{y}$ sus variaciones intra-específicas (e.g. Oldeman, 
1989). Entre tales variaciones se encuentran aquellas que resultan de cambios cualitativos en los componentes estructurales de los individuos de una especie durante su ontogenia; a dichas variaciones se las conoce como gradientes morfogenéticos (Barthélémy \& Caraglio, 2007).

En plantas leñosas con crecimiento rítmico, es posible reconocer el gradiente morfogenético propio de la especie en las variaciones de las características morfológicas de las unidades de crecimiento (UC; plural UCs) de sus ejes, definiéndose a una UC como todo segmento o porción de un eje alargado en forma ininterrumpida (Barthélémy et al., 1997; Sabatier \& Barthélémy, 1999; Passo et al., 2002; Ishihara \& Kikuzawa, 2009; Stecconi et al., 2010). Entre estas características morfológicas se incluyen: longitud, diámetro, número de nudos, filotaxis, orientación espacial y patrón de ramificación (Guérard et al., 2001; Passo et al., 2002; Umeki \& Seino, 2003; Heuret et al., 2006; Guédon et al., 2007; Calabria \& Puntieri, 2008; Chaubert-Pereira et al., 2009). El patrón de ramificación de los ejes, es reconocido como uno de los componentes de la arquitectura de una planta que poseen mayor valor adaptativo (Tomlinson, 1987), aunque su complejidad morfogenética plantea más dificultades en su evaluación y cuantificación que las restantes características mencionadas. En toda planta, un sistema ramificado puede caracterizarse tanto por las distribuciones espacial y temporal de las ramas como por la diferenciación de éstas respecto del eje que las porta (Suzuki \& Suzuki, 2009). Un nivel de diferenciación alto supone la existencia de ejes especializados en funciones determinadas (Puntieri et al., 2003; Suzuki \& Suzuki, 2009). En estudios ecológicos es frecuente que los sistemas ramificados con alto nivel de diferenciación entre categorías de ejes se describan como sistemas ramificados jerárquicos (Pickett \& Kempf, 1980; Steingraeber, 1982; Stevens \& Perkins, 1992; Fig. 1). El grado de desarrollo jerárquico de un sistema de ramificación puede analizarse en base a la orientación de crecimiento y al tamaño (medido, por ejemplo, en diámetro) de su eje principal respecto de las ramas derivadas de éste; cuanto más similares son el eje principal y sus ramas en cuanto a orientación y tamaño, menos jerárquico es el sistema ramificado.

Plantas de una misma especie pueden responder a diferentes condiciones ambientales mediante

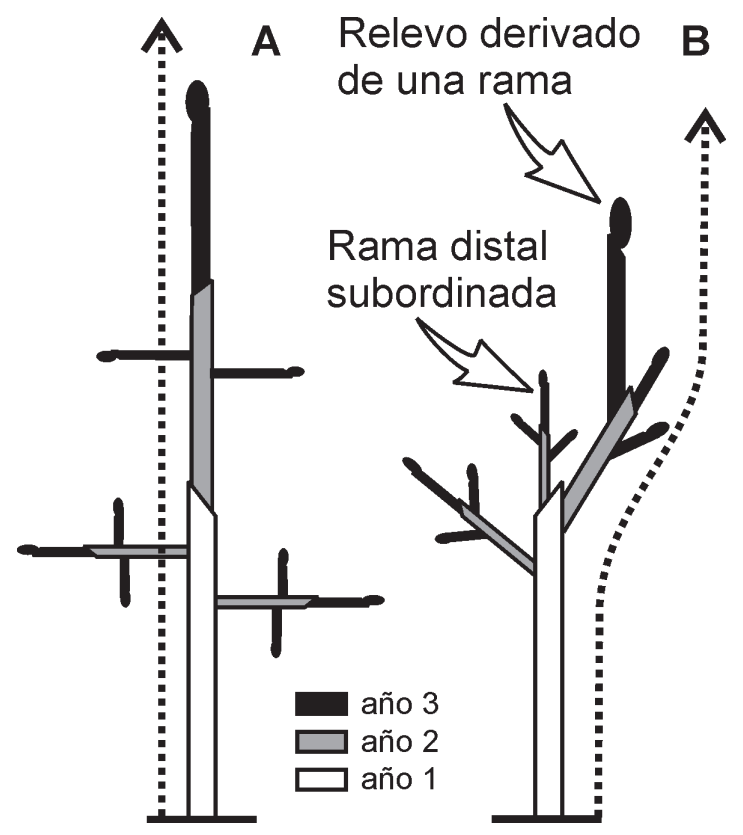

Fig. 1. Esquemas de sistemas de ramificación enteramente simpodiales (por muerte del ápice de cada unidad de crecimiento) con diferente estructura jerárquica, ambos constituidos por unidades de crecimiento correspondientes a tres años. A: Sistema marcadamente jerárquico, con las ramas laterales claramente divergentes y de tamaño menor al del eje portador (acompañado por una flecha de trazos que indica la dirección de crecimiento). B: Sistema poco jerárquico, en el cual cada unidad de crecimiento del eje principal desarrolla ramas laterales escasamente divergentes y de tamaños semejantes a las del portador; en este caso, el eje principal (flecha de trazos) adopta un desarrollo más sinuoso por la posición de la rama que actúa como relevo en cada año. En (B) la rama más distal derivada del año 1 continúa la línea de crecimiento del portador, pero queda subordinada por el mayor crecimiento de una rama más proximal (ver texto por detalles).

cambios en la expresión del gradiente morfogenético de esa especie (Barthélémy \& Caraglio, 2007; Stecconi et al., 2010). La disponibilidad de luz es uno de los factores que varían en forma más notable entre micrositios dentro de una comunidad vegetal, y la capacidad de una especie para tolerar diferentes niveles de sombra podría estar ligada a sus atributos arquitecturales (Horn, 1971; Küppers, 1994). Las plantas tolerantes a la sombra poseen adaptaciones fisiológicas, anatómicas, morfológicas 


\section{J. Puntieri et al. - Patrones de ramificación en Nothofagus}

y/o arquitecturales que les permiten persistir en esos ambientes por períodos prolongados (e.g. Valladares \& Niinemets, 2007; Valladares and Niinemets, 2008; Coste et al., 2009). En otras plantas, por el contrario, han evolucionado respuestas tendientes a evitar los ambientes sombreados (e.g. Henry \& Aarssen, 1997; Schmitt et al., 2003; Franklin \& Whitelam, 2005; Charles-Dominique et al., 2010). Entre las características arquitecturales de las plantas leñosas que podrían estar sujetas a las condiciones lumínicas se encuentra el grado de jerarquía de sus sistemas de ramificación. Algunos estudios acerca de los efectos de la sombra en el desarrollo de las plantas leñosas sugieren que, en especies de baja tolerancia a la sombra, el sombreo de un eje afecta negativamente tanto al número de ramas como al tamaño de las mismas respecto del tamaño del eje que las porta, desarrollándose un sistema ramificado más jerárquico (Steingraeber et al., 1979; Pickett \& Kempf, 1980; Luken et al., 1995; Day, 1998; Valladares \& Niinemets, 2008; Valladares et al., 2011). Sin embargo, para una especie muy tolerante a la sombra se han encontrado sistemas ramificados más jerárquicos en sotobosque que en zonas abiertas (CharlesDominique et al., 2012). Este contraste entre estudios podría explicarse por diferencias en la forma de evaluación del grado de desarrollo jerárquico de los sistemas ramificados; además, sólo en el último de los estudios citados se tomó en cuenta el gradiente morfogenético de la especie analizada. Las modificaciones en la estructura de los ejes de las plantas bajo diferentes condiciones lumínicas pueden contribuir a explicar diferencias de distribución entre especies emparentadas (Horn, 1971; Delagrange et al., 2006; Seiwa et al., 2006; Niklas 2007; Valladares \& Niinemets, 2007).

Las especies arbóreas patagónicas Nothofagus alpina (Poepp. \& Endl.) Oerst. (= N. nervosa (Phil.) Krasser) y N. obliqua (Mirb.) Oerst. (Familia Nothofagaceae) están estrechamente emparentadas y comparten atributos morfo-arquitecturales y ecológicos (Donoso, 2006a, b; Puntieri \& Ghirardi, 2010). Por otro lado, sus diferencias arquitecturales han sido poco estudiadas (ver Torres et al., 2009). Si bien la tolerancia a la sombra de ambas especies es descripta como intermedia a baja, $N$. alpina es considerada más tolerante a la sombra que $N$. obliqua debido a la respuesta de sus aparatos fotosintéticos a diferentes radiaciones incidentes
(Read \& Hill, 1985; Donoso, 2006a, b). No obstante, pueden encontrarse ejemplares juveniles de ambas especies creciendo bajo el dosel de bosques mixtos de Nothofagus (en el Parque Nacional Lanín) y ejemplares de tamaños similares en vivero (e.g. INTA Bariloche). Los objetivos del presente estudio fueron determinar si el crecimiento y la ramificación de las ramas principales de estas especies difiere al desarrollarse los árboles bajo diferentes condiciones lumínicas (en comunidades naturales y en vivero) y si existen diferencias interespecíficas en el desarrollo de las ramas principales, luego de incorporar el componente de variación morfogenética. En base a la mayoría de los estudios disponibles sobre esta temática, se esperaría que, con el incremento en el nivel de sombreo, las ramas de los árboles de estas especies desarrollaran UCs más cortas y delgadas y menos ramificadas, y que exhibieran un desarrollo más jerárquico; también cabría suponer que los efectos de la sombra fueran más notables en $N$. obliqua, por su menor tolerancia a la sombra. Los resultados permitirían determinar si los atributos arquitecturales de estas especies guardan relación con sus afinidades ecológicas y, en definitiva, con sus distribuciones geográficas.

\section{Materiales y Métodos}

\section{Estructura básica de las ramas principales en Nothofagus}

En los árboles juveniles-adultos de Nothofagus, las ramas principales (RP; plural RPs) son ejes derivados del tronco que inician su desarrollo en forma horizontal u oblicua, tienen larga vida (por lo general más de 20 años), y portan ramas menores (Fig. 2A; Puntieri et al., 2003). Cada UC de una RP desarrolla, en el año siguiente al de su extensión, un conjunto de ramas laterales cuyo tamaño aumenta hacia el ápice de la UC portadora (Fig. 2B). En estas especies es muy frecuente que se produzca la muerte del ápice de una UC al finalizar el alargamiento de la misma, de manera que el sistema de ramificación de las RPs es predominantemente simpodial (Barthélémy \& Caraglio, 2007). El crecimiento longitudinal de una RP luego de la muerte del ápice de una UC se produce por el desarrollo, en el período de crecimiento siguiente, de una UC relevo derivada de una de las ramas de la UC portadora (Fig. 1A; Puntieri \& Ghirardi, 2010). 


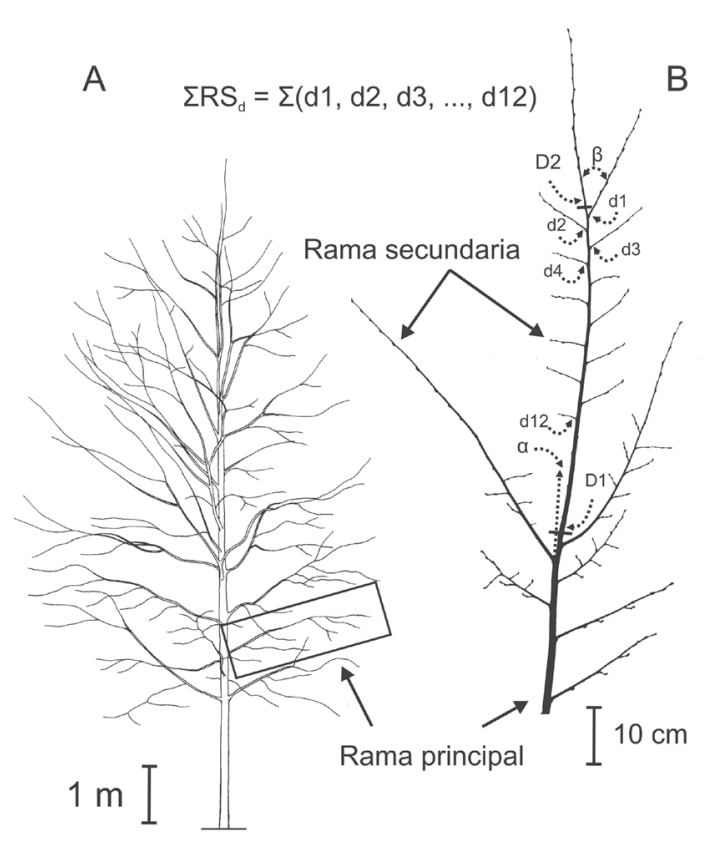

Fig. 2. A: llustración de un árbol de $N$. alpina como los seleccionados para este estudio. Una rama principal baja ha sido marcada con un rectángulo. B: Vista superior de un segmento de una rama principal de $N$. obliqua con tres unidades de crecimiento, la más proximal de ellas incompleta. Los límites entre unidades de crecimiento están marcados con líneas gruesas y cortas. Se indican los ángulos formados entre dos unidades de crecimiento sucesivas de la rama principal, $\alpha$, y entre una unidad de crecimiento de una rama principal y una de sus ramas secundarias, $\beta$. Los puntos de medición de los diámetros de las unidades de crecimiento (D para las ramas principales y d para las ramas secundarias) se han indicado con flechas punteadas. Se indica la fórmula para el cálculo de la suma de los diámetros de las ramas secundarias $\left(\sum R S_{d}\right)$ derivadas de una de las unidades de crecimiento de la rama principal (D1). Ver más detalles en el texto.

Puede ocurrir, con baja frecuencia, el desarrollo de un relevo axilar por muerte del ápice de una UC sin que medie un período de reposo invernal (rebrote intra-anual). Las restantes ramas derivadas de la misma UC portadora se incluyen en otra categoría de ejes denominada rama secundaria (RS; plural RSs), aunque a menudo una o dos de estas ramas divergen en forma poco marcada de la RP, constituyendo ejes co-dominantes.

\section{Sitios y árboles de muestreo}

Se seleccionaron árboles de $N$. alpina y $N$. obliqua desarrollados en poblaciones naturales localizadas en el área de Nonthué, al norte del Lago Lácar, en el Parque Nacional Lanín ( $40^{\circ} 08^{\prime}$

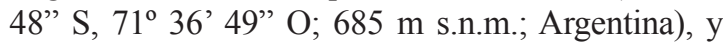
árboles desarrollados en el Vivero Forestal del INTA

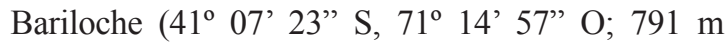
s.n.m.; Argentina).

Los árboles de Nonthué habitan en un bosque bien preservado de $N$. alpina, $N$. obliqua y $N$. dombeyi, cuyo dosel está formado por árboles de más de $1 \mathrm{~m}$ de diámetro de tronco, alrededor de 25 $\mathrm{m}$ de altura y más de 50 años de edad. La dinámica de este bosque está mayormente dictada por procesos naturales: la regeneración de las especies arbóreas tiene lugar principalmente debajo de los claros que se forman en el dosel, aunque es posible encontrar árboles con diámetro del tronco menor a $30 \mathrm{~cm}$ debajo del dosel. La evaluación de la edad del tronco de los árboles desarrollados debajo del dosel indicó que los mismos son mucho menores en edad que aquellos que componen el dosel. Sobre las banquinas a lo largo de la sección de la ruta nacional 48 que cruza esta área, se han generado condiciones para el desarrollo de una franja de vegetación desprovista de dosel alto, constituyéndose un sitio adecuado para la regeneración de las especies de Nothofagus; allí se encuentran árboles de edades variadas expuestos a niveles de luminosidad altos. Se seleccionaron diez árboles de $N$. alpina y diez de $N$. obliqua de entre 5 y $10 \mathrm{~m}$ de altura desarrollados debajo del dosel del bosque dentro de un área de aproximadamente 20 ha. La densidad de estos árboles de sotobosque era baja y los árboles seleccionados estaban separados entre sí y de otros árboles del sotobosque por más de $10 \mathrm{~m}$. Adicionalmente, se seleccionaron diez árboles de cada especie desarrollados lo largo de $1 \mathrm{~km}$ de la banquina de la ruta 48. Si bien la densidad de árboles de la banquina era alta en algunos sectores (hasta 1 árbol por $\mathrm{m}^{2}$ ), todos los árboles seleccionados se encontraban libres de interferencia por otros árboles. Las edades de estos árboles se determinaron en base al conteo de UCs de las ramas por criterios morfológicos (Puntieri \& Ghirardi, 2010; Tabla 1).

En el vivero forestal se seleccionaron 21 árboles de $N$. alpina y cinco de $N$. obliqua comparables en tamaño y estructura con aquellos seleccionados en Nonthué. Estos árboles se encontraban dispuestos en hileras, distanciados a no menos de $3 \mathrm{~m}$. La edad 


\section{J. Puntieri et al. - Patrones de ramificación en Nothofagus}

de los árboles seleccionados del vivero forestal fue levemente diferente entre las dos especies (Tabla 1), aunque todos ellos habían sido plantados en el vivero 18 años antes del estudio. Una hilera de árboles de Pseudotsuga menziesii se encontraba al noroeste de los Nothofagus del vivero, provocando cierto grado de sombreo.

Se juzgó que todos los árboles seleccionados se encontraban en una fase de desarrollo similar al momento del muestreo: tenían un tronco vertical o algo inclinado bien definido, de entre 5 y $10 \mathrm{~m}$ de altura y menos de $30 \mathrm{~cm}$ de diámetro basal y RPs horizontales. Algunos de los árboles seleccionados habían iniciado la producción de flores en baja cantidad. En base a diez fotografías hemisféricas, tomadas a $2 \mathrm{~m}$ por encima del nivel del suelo en cada área de muestreo, y al análisis de esa imágenes con el programa Image J, se determinó que los tres sitios de muestreo presentaban distintas condiciones lumínicas: alta en la banquina, intermedia en el vivero y baja en el sotobosque (Tabla 1). Los suelos de los tres sitios de muestreo son profundos y se componen principalmente de sedimentos glaciofluviales y cenizas volcánicas, como es típico de la región noroccidental de la Patagonia (Scoppa 1998). Los datos bibliográficos disponibles acerca de las condiciones climáticas indican que las temperaturas medias y las precipitaciones son semejantes en Nonthué y en el vivero forestal (Conti, 1998). Es decir que las observaciones realizadas y la información bibliográfica disponible indican que las tres áreas de muestreo difieren especialmente en la disponibilidad de luz y son similares en cuanto a sus atributos edáficos y climáticos.

\section{Ramas principales muestreadas}

Entre febrero y marzo de 2009 se cortó de cada árbol seleccionado, cerca del sitio de origen sobre el tronco, una RP ubicada entre 1,5 y $4,0 \mathrm{~m}$ de altura sobre el suelo. En base al conteo de los anillos de crecimiento radial, se evaluó que las RPs cortadas habían derivado del tronco entre 11 y 21 años antes del muestreo. Se evitaron aquellas RPs con signos de daño severo provocado por factores exógenos. En el caso de los árboles de la banquina, sólo se seleccionaron ramas orientadas hacia la ruta. En el laboratorio, se identificaron las UCs de cada una de estas RPs en base a las cicatrices dejadas por los catafilos basales correspondientes a las yemas de invierno y al conteo de anillos de crecimiento
Tabla 1. Datos sobre los árboles y los sitios de

muestreo. Se indica la media ( \pm 1 error estándar)

de la altura, el diámetro basal y la edad de los

árboles, y el porcentaje de cobertura del dosel

(cada media estimada a partir de 10 imágenes

digitales) en el sotobosque de un bosque de

Nothofagus, a lo largo de una banquina y en un vivero forestal.

\begin{tabular}{|lccc|}
\hline & \multicolumn{3}{c}{ Sitio de muestreo } \\
& Sotobosque & Vivero & Banquina \\
Altura árbol (m) & & & \\
$N$. alpina & $7,3 \pm 0,6$ & $7,1 \pm 0,4$ & $6,2 \pm 0,5$ \\
$N$. obliqua & $6,7 \pm 0,4$ & $8,0 \pm 0,6$ & $6,6 \pm 0,5$ \\
Diám. tronco (cm) & & & \\
$N$. alpina & $9,5 \pm 0,9$ & $13,8 \pm 0,7$ & $9,8 \pm 0,9$ \\
$N$. obliqua & $8,4 \pm 0,6$ & $19,6 \pm 1,0$ & $12,9 \pm 0,7$ \\
Edad árbol (años) & & & \\
$N$. alpina & $23 \pm 1,2$ & 20 & $17 \pm 1,2$ \\
$N$. obliqua & $25 \pm 1,6$ & 23 & $29 \pm 1,7$ \\
Cobertura (\%) & $88,9 \pm 0,7$ & $65,7 \pm 3,3$ & $38,9 \pm 1,8$ \\
\hline
\end{tabular}

radial en cortes transversales del tallo (Barthélémy et al., 1999). Sólo en dos casos se identificaron UCs que incluyeron un rebrote intra-anual. Se midieron el diámetro basal (con precisión de $0,1 \mathrm{~mm}$ ) y la longitud (precisión de $0,1 \mathrm{~cm}$ ) y se contó el número de hojas de cada UC (Fig. 2B). Las hojas se contaron a partir de la observación bajo lupa de las cicatrices dejadas por los pecíolos de las hojas en la superficie del tallo. En Nothofagus, cada UC posee una serie de catafilos basales que corresponden, invariablemente, con entrenudos cortos, y que pueden permanecer en pie hasta el final de la estación de crecimiento en que se alargó la UC correspondiente. Al pasar los años desde el alargamiento de una UC, el conteo de sus catafilos se vuelve sumamente difícil o incluso imposible, ya que para ello es necesario recurrir a las cicatrices dejadas por los catafilos en la superficie del tallo, las que no siempre se identifican claramente. Por esta razón, los catafilos no fueron tomados en cuenta en la evaluación del número de hojas de las UCs. Se registró la presencia/ausencia de RS en cada nudo de cada RP y el diámetro basal de cada RS encontrada. Las RSs muertas no fueron incluidas en las mediciones. Se calculó la suma de los diámetros de las RSs $\left(\Sigma \mathrm{RS}_{\mathrm{d}}\right)$ para cada UC de las RPs.

Mediante el uso de un transportador, se registraron: el ángulo formado entre dos UCs sucesivas de cada $\mathrm{RP}(\alpha)$, y el ángulo formado entre cada RS y su RP portadora ( $\beta$; Fig. 2 B). La mayoría de las RPs y las RSs se orientaron lateralmente respecto de sus respectivas UCs portadoras, disponiéndose todas en un plano aproximadamente horizontal. 
Con mucha menor frecuencia se observaron RSs orientadas hacia arriba o hacia abajo (no se presenta esta información numérica, pero se la puede solicitar al primer autor). Para cada RP se registró la información acerca de los ángulos $\alpha$ y $\beta$ para el mayor número posible de UCs (entre seis y $21 \mathrm{UCs}$ ). En muchos casos, la presencia de líquenes en el súber impidió la identificación de límites entre UCs y el conteo de hojas.

Evaluación de la estructura jerárquica de los sistemas ramificados

Se evaluó la estructura jerárquica de los sistemas ramificados mediante: (1) el cociente entre el diámetro del tallo de las RSs y el de la UC correspondiente a la RP portadora, (2) el ángulo entre UCs sucesivas de la RP $(\alpha)$, y (3) el ángulo formado entre las RSs y la RP portadora $(\beta)$. Considerando los parámetros mencionados anteriormente, a medida que el sistema de ramificación se vuelve más jerárquico debería: (1) disminuir la relación entre el diámetro de las RSs y el diámetro de la RP, (2) disminuir el ángulo $\alpha, y$ (3) aumentar el ángulo $\beta$ (Fig. 1A). En contraste, en un sistema ramificado poco jerárquico, las RSs se deberían asemejarse a las RPs tanto en diámetro como en dirección de crecimiento, en tanto que las UCs de las RPs deberían presentar una trayectoria más sinuosa que en un sistema altamente jerárquico (Fig. 1B).

\section{Análisis de datos}

Se analizaron cuantitativamente las variaciones en el diámetro, la longitud, el número de hojas, el número de RSs y la $\Sigma \mathrm{RS}_{\mathrm{d}}$ de las UCs según el año calendario en que éstas se desarrollaron. Los nudos de cada UC se numeraron según su posición desde el extremo distal al extremo proximal de la UC. Para cada año de desarrollo de la UC, especie y sitio de crecimiento, se calculó la proporción de nudos de cada posición que presentaron una RS.

Se analizaron estadísticamente los siguientes aspectos del desarrollo de las RPs: (1) los atributos arquitecturales de sistemas ramificados completos y de UCs recientes, y el desarrollo jerárquico de las RPs medido (2) por el diámetro de las RSs relativo al diámetro de la UC portadora, y (3) por los ángulos formados entre UCs sucesivas de las RPs $(\alpha)$ y entre RSs y RPs $(\beta)$.

(1) Se evaluaron los siguientes atributos descriptores de RPs enteras (es decir, desde su derivación desde el tronco): el diámetro basal de la RP, la suma de las longitudes y los números de nudos de las UCs de la RP y $\Sigma \mathrm{RS}_{\mathrm{d}}$. Se compararon los siguientes atributos de las UCs extendidas en la estación de crecimiento inmediatamente precedente a la del muestreo (o sea 2008-2009): diámetro, longitud, número de hojas, la relación longitud/diámetro (denominada esbeltez del tallo) y la relación longitud/número de hojas (o sea longitud media por entrenudo). Para cada una de estas variables se aplicó análisis de covariancia (ANCOVA) de dos vías (procedimiento GLM para diseños no balanceados; Zar, 1999), incluyendo sitio de muestreo (sotobosque, banquina y vivero) y especie ( $N$. alpina y $N$. obliqua) como factores y el número de años desde la incepción de la rama a partir del tronco (edad de la rama) como covariable. A fin de evaluar el grado de ramificación de las UCs recientes y el tamaño de sus ramas, se compararon el número de RSs y $\Sigma \mathrm{RS}_{\mathrm{d}}$ para UCs de las RPs extendidas en la estación de crecimiento 2007-2008. Para estas dos variables, se aplicó ANCOVA según el modelo ya descripto, pero incluyendo el número de hojas de la UC de 2007-2008 como covariable ya que, en Nothofagus, el número de ramas por UC está linealmente relacionado con el número de hojas de la UC (Puntieri et al., 2003).

(2) Se evaluó el desarrollo relativo de las RSs derivadas de las RPs a dos niveles. A nivel de RPs enteras, se calculó la relación entre $\Sigma \mathrm{RS}_{\mathrm{d}}$, incluyendo a todas las RSs, y el diámetro de la RP en su extremo proximal (unión con el tronco del árbol). A nivel de la UC ramificada más distal de las RPs, se calculó la relación entre $\Sigma \mathrm{RS}_{\mathrm{d}}$ correspondiente a la estación 2008-2009, y el diámetro de la RP en la base de la UC extendida en 2007-2008 (o sea la UC portadora). Para determinar si el tamaño relativo de las RSs varió entre estos dos niveles de observación de las RPs, se compararon los tamaños relativos de las RSs a estos dos niveles por medio de ANCOVA de tres vías, incluyendo sitio, especie y nivel de observación (ramificación reciente o total) como factores, y la edad de la RP como covariable. Todas las variables descriptas hasta ahora cumplieron los pre-requisitos de normalidad (prueba de Kolmogorov-Smirnov) y homocedasticidad (prueba de Levene) necesarios para aplicar ANCOVA, ya sea sobre los datos no transformados o después de aplicar transformaciones logarítmicas.

(3) La distribución de los ángulos $\alpha$ se desvió 
significativamente de la normalidad aún después de aplicar transformaciones. Por esta razón, dicha variable fue categorizada a fin de posibilitar su comparación: $\alpha=0^{\circ}$ (una UC sigue la línea de crecimiento de la UC anterior), $0^{\circ}<\alpha<50^{\circ}$ (dos UCs sucesivas forman un ángulo cerrado) y $\alpha \geq$ $50^{\circ}$ (dos UCs sucesivas forman un ángulo abierto). Los porcentajes de UCs en cada una de estas categorías fueron comparados entre $N$. alpina y $N$. obliqua, considerando los tres sitios combinados o separándolos, por medio de pruebas de Chicuadrado $\left(\chi^{2}\right)$; también se compararon los tres sitios después de combinar los datos de ambas especies. La distribución de los ángulos $\beta$ también se desvió significativamente de la normalidad aún después de aplicar transformaciones. Al observar estos datos, quedó en evidencia que esta desviación se debió a que en 52 de las 1263 RSs medidas, la RS siguió la línea de crecimiento de la UC portadora (o sea $\beta=0$ ). Este es un caso particular en el cual una RS se origina como un relevo de la RP pero pierde vigor y queda subordinada a otra rama que continúa el desarrollo longitudinal del eje portador (Fig. 1B). La distribución de $\beta>0$ se ajustó a la normalidad, de modo que estos datos pudieron ser comparados mediante ANCOVA de dos vías, incluyendo sitio y especie como factores, y posición de la RS sobre la UC portadora y año de extensión de la UC de la RP como covariables. La proporción de RSs con $\beta=0$ se comparó entre especies (para todos los sitios combinados) y entre sitios (para ambas especies combinadas) con pruebas de $\chi^{2}$.

Todas las comparaciones estadísticas se realizaron con el programa R (Venables et al., 2010), empleando un nivel de significación de 0,05 .

\section{Resultados}

Atributos arquitecturales de las ramas principales

Las comparaciones estadísticas de RPs completas, luego de incluir el efecto de la edad de la RP, indicaron diferencias significativas entre sitios para todas las variables consideradas: diámetro, longitud, número de hojas y $\Sigma \mathrm{RS}_{\mathrm{d}}$ de la $\mathrm{RP}$ (vivero > banquina $>$ sotobosque; Tabla 2). Con respecto a la longitud y al número de hojas de las RPs completas, las diferencias entre los árboles del sotobosque y aquellos de los otros dos sitios fueron más notables para $N$. obliqua que para $N$. alpina, haciendo significativa la interacción entre los factores "sitio de muestreo" y "especie" (Tabla 2; Fig. 3B, C). El efecto de la edad de la RP fue significativo para el diámetro y, en particular, para el número de hojas (Tabla 2).

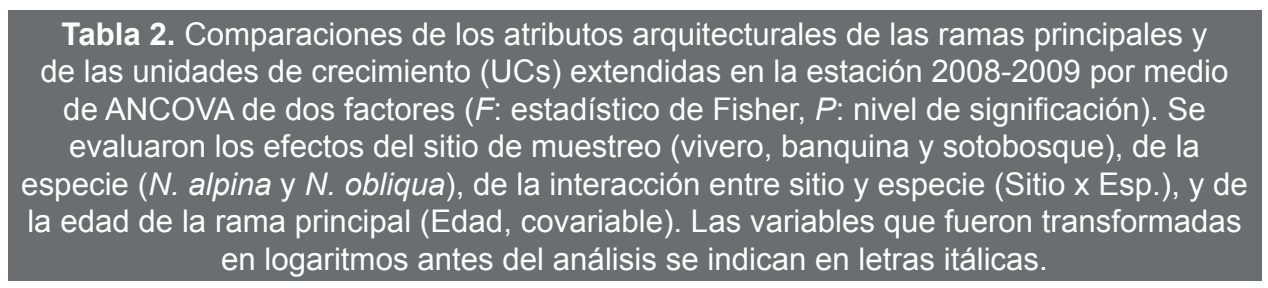

\begin{tabular}{|c|c|c|c|c|c|c|c|c|}
\hline \multirow[b]{2}{*}{ Rama principal } & \multicolumn{2}{|c|}{ Sitio } & \multicolumn{2}{|c|}{ Especie } & \multicolumn{2}{|c|}{ Sitio x Esp. } & \multicolumn{2}{|c|}{ Edad } \\
\hline & $F$ & $p$ & $F$ & $p$ & $F$ & $p$ & $F$ & $p$ \\
\hline Diámetro ${ }^{1}$ & 62,5 & $* * *$ & 0,4 & ns & 2,9 & ns & 4,4 & * \\
\hline Longitud $^{2}$ & 46,7 & $* * *$ & 3,7 & $n s$ & 5,1 & $\star *$ & 3,1 & $n s$ \\
\hline $\mathrm{N}^{0}$ de hojas ${ }^{3}$ & 33,1 & $* * *$ & 101,3 & $* * *$ & 6,6 & ** & 45,5 & $* * *$ \\
\hline$\sum \mathrm{RS}_{\mathrm{d}}^{4}$ & 23,2 & $* * *$ & 0,3 & ns & 0,9 & ns & 1,4 & ns \\
\hline UC de 2008-2009 & & & & & & & & \\
\hline Diámetro 5 & 8,6 & ** & 9,6 & ** & 2,2 & ns & 0,0 & ns \\
\hline Longitud $^{6}$ & 3,1 & $n s$ & 2,0 & $n s$ & 2,7 & $n s$ & 0,1 & $n s$ \\
\hline $\mathrm{N}^{0}$ de hojas ${ }^{7}$ & 3,1 & ns & 11,5 & $* *$ & 3,7 & * & 0,0 & $n s$ \\
\hline Esbeltez $^{8}$ & 1,4 & $n s$ & 9,8 & ** & 1,7 & $n s$ & 0,3 & $n s$ \\
\hline Long. entrenudos ${ }^{9}$ & 2,4 & $n s$ & 0,2 & ns & 1,7 & $n s$ & 0,4 & $n s$ \\
\hline
\end{tabular}

Referencias: ${ }^{1}$ Diámetro de la rama principal en su extremo proximal; ${ }^{2}$ Longitud de la rama principal completa; ${ }^{3}$ Número de hojas desarrolladas por una rama principal; ${ }^{4}$ Suma de los diámetros de todas las ramas secundarias derivadas de una rama principal; ${ }^{5}$ Diámetro del tallo de la UC extendida en 2008-2009 en su extremo proximal; ${ }^{6}$ Longitud del tallo de la UC extendida en 2008-2009; ${ }^{7}$ Número de hojas de la UC extendida en 2008-2009; ${ }^{8}$ Cociente entre la longitud y el diámetro proximal de la UC extendida en 2008-2009; ${ }^{9}$ Cociente entre la longitud del tallo y el número de hojas de la UC extendida en 2008-2009; ${ }^{* *} P<0,001$, ** $P<0,01,{ }^{*} P<0.05$, ns $P>0,05$. 
El incremento del diámetro de las UCs de las RPs al aumentar la edad de la UC (por efecto del crecimiento cambial) fue máximo en los árboles del vivero, intermedio en los de la banquina y mínimo en los del sotobosque (Fig. 3A). La longitud y el número de hojas de esas UCs fluctuaron notablemente $\mathrm{y}$, en términos generales, fueron mayores para los árboles del vivero que para los de la banquina, y mayores en éstos que en los árboles del sotobosque para cada especie y año de extensión de la UC (Fig. 3B, C). El número de RSs por UC de las RPs (excepto para las UCs más recientes, extendidas en 2008-2009, que todavía no habían desarrollado ramas) tendió a decrecer con el aumento en la edad de las UCs en el caso de árboles del vivero y de la banquina, y fue relativamente constante y bajo (ninguna, una o dos) para los árboles del sotobosque (Fig. 4A). En los árboles del vivero y de la banquina, la suma de los diámetros de las RSs $\left(\Sigma \mathrm{RS}_{\mathrm{d}}\right)$ aumentó desde las UCs extendidas en 2008-2009 hasta las UCs extendidas en los dos a seis años previos; UCs extendidas entre siete y 16 años antes del muestreo (2002-1993) tendieron a presentar $\Sigma \mathrm{RS}_{\mathrm{d}}$ más bajas (Fig. 4B). Las UC de las RPs de los árboles del sotobosque tuvieron $\Sigma \mathrm{RS}_{\mathrm{d}}$ menores y menos variables.

Exceptuando a las UCs de las RPs del año más reciente (que no estaban ramificadas), la mayoría de las UCs de las RPs de todos los años presentaron RSs en los dos nudos distales. La proporción de nudos portadores de RSs decreció a medida que se consideraron posiciones más proximales en las UCs, tendencia que se acrecentó en UCs de más años (Fig. 4C, D). La presencia de RSs en las UCs extendidas en 2007-2008 fue más frecuente, para cada posición, en árboles del vivero y de la banquina que en los del sotobosque. Las diferencias entre sitios en cuanto a la presencia de RSs fueron menores para UCs de mayor edad. Las UCs extendidas en 2007-2008 y en 2001-2002 fueron más similares en cuanto a la presencia de RSs en los árboles del sotobosque que en los de los otros dos sitios (Fig. 4C, D).

UCs extendidas en 2008-2009 difirieron en diámetro siguiendo el gradiente: vivero $>$ banquina $>$ sotobosque, y presentaron mayor diámetro y menor número de hojas en $N$. alpina que en $N$. obliqua (Tabla 2; Fig. 3A, C). El número de hojas de las UCs de 2008-2009 fue mayor en los árboles de $N$. obliqua de banquina que en los del vivero,
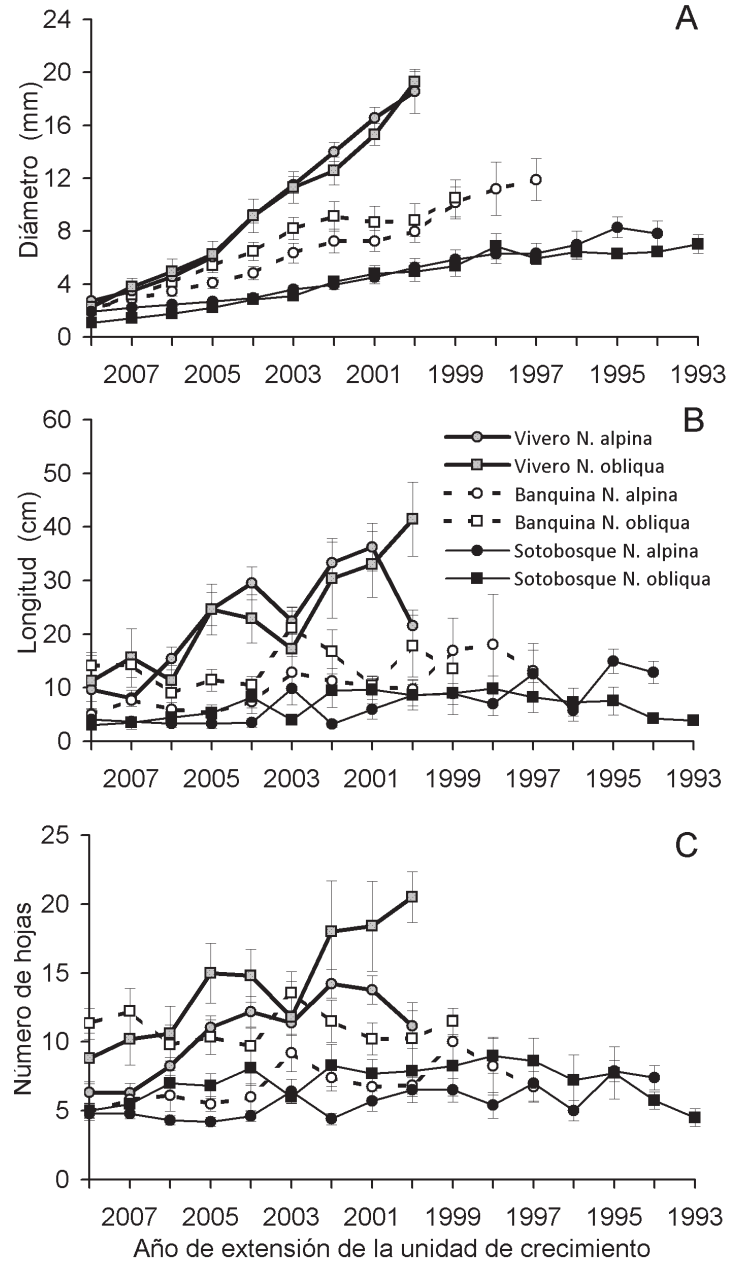

Fig. 3. Unidades de crecimiento de las ramas principales extendidas en años sucesivos para árboles de $N$. alpina (círculos) y $N$. obliqua (cuadrados) desarrollados en vivero, banquina $y$ sotobosque. A: Promedios ( $\pm 1 \mathrm{EE})$ del diámetro. B: Longitud. C: Número de nudos.

mientras que lo contrario se observó para los árboles de $N$. alpina, resultando en una interacción especie $\mathrm{x}$ sitio significativa para esa variable (Tabla 2; Fig. 3C). Los promedios de longitud, longitud media por entrenudo y esbeltez de las UCs extendidas en 20082009 tendieron a ser menores para los árboles del sotobosque que para los de los restantes dos sitios (Fig. 3B, Fig. 5), aunque los análisis estadísticos sobre datos transformados logarítmicamente no revelaron diferencias significativas entre sitios para ninguna de estas variables (Tabla 2). La longitud de 

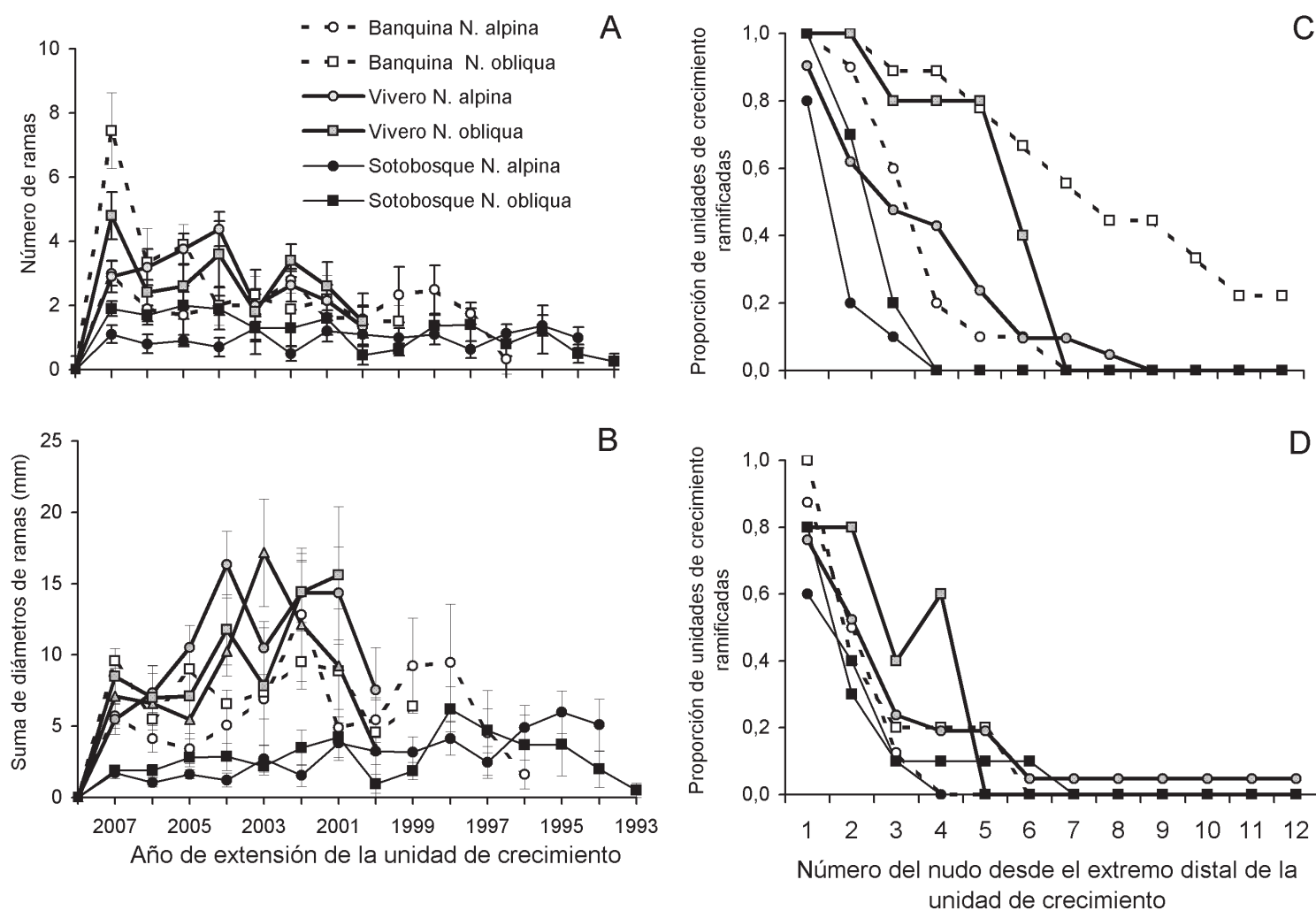

Fig. 4. A y B: Promedios ( $\pm 1 \mathrm{EE})$ del número de ramas $(A)$ y de la suma de los diámetros de las ramas (B) derivadas de unidades de crecimiento de las ramas principales extendidas en años sucesivos, para árboles de $N$. alpina (círculos) y $N$. obliqua (cuadrados) desarrollados en vivero, banquina y sotobosque. C y D: Proporciones de nudos de las ramas principales (numerados desde el nudo más distal de cada unidad de crecimiento) portadores de ramas vivas para unidades de crecimiento extendidas en las estaciones de crecimiento 2007-2008 (C) y 2001-2002 (D).

las UCs y la longitud media por entrenudo fueron similares entre especies, y la esbeltez de las UCs fue mayor para N. obliqua que para N. alpina (Tabla 2; Fig. 5). Tanto el número de RSs como $\Sigma \mathrm{RS}_{\mathrm{d}}$ de las UCs extendidas en 2007-2008 variaron entre sitios ([vivero = banquina $]>$ sotobosque; Fig. 4B); $\Sigma \mathrm{RS}_{\mathrm{d}}$ también varió entre especies $(N$. obliqua $>$ N. alpina; Tabla 3). El número de hojas de la UC portadora tuvo una influencia directa significativa sobre el número de RSs y $\Sigma \mathrm{RS}_{\mathrm{d}}$ (Tabla 3 ).

\section{Estructura jerárquica de las ramas principales}

La relación entre $\Sigma \mathrm{RS}_{\mathrm{d}}$ y el diámetro de la RP no difirió entre sitios, fue mayor para $N$. obliqua que para $N$. alpina, y menor para las UCs extendidas en 2007-2008 que para aquellas de la RP completa (Tabla 3; Fig. 6). Ni las interacciones entre estos factores, ni la edad de la RP afectaron a esta relación.

Para la mayoría de las RPs muestreadas, UCs sucesivas siguieron aproximadamente una línea de desarrollo $\left(\alpha<10^{\circ}\right)$ o se desviaron en ángulos menores de $50^{\circ}$ (Fig. 7A). La comparación entre sitios (combinando ambas especies) indicó una diferencia no significativa en $\alpha\left(\chi^{2}=8,7 ; p=0,066\right)$. Al comparar $N$. alpina y $N$. obliqua, no se encontraron diferencias en las distribuciones de $\alpha$, ya sea al combinar los datos de los tres sitios $\left(\chi^{2}=4,9\right.$; $p=0,086$ ), o al considerar cada uno de ellos por separado $\left(\chi^{2}=0,1,4,4\right.$ y 2,9 , respectivamente para banquina, vivero y sotobosque; $p>0,10$ ).

Las RSs formaron ángulos de entre $0^{\circ}$ y $140^{\circ}$ con sus UCs portadoras $(\beta)$. La media de $\beta$ (descontando los casos de $\beta=0$ ) fue de alrededor de 


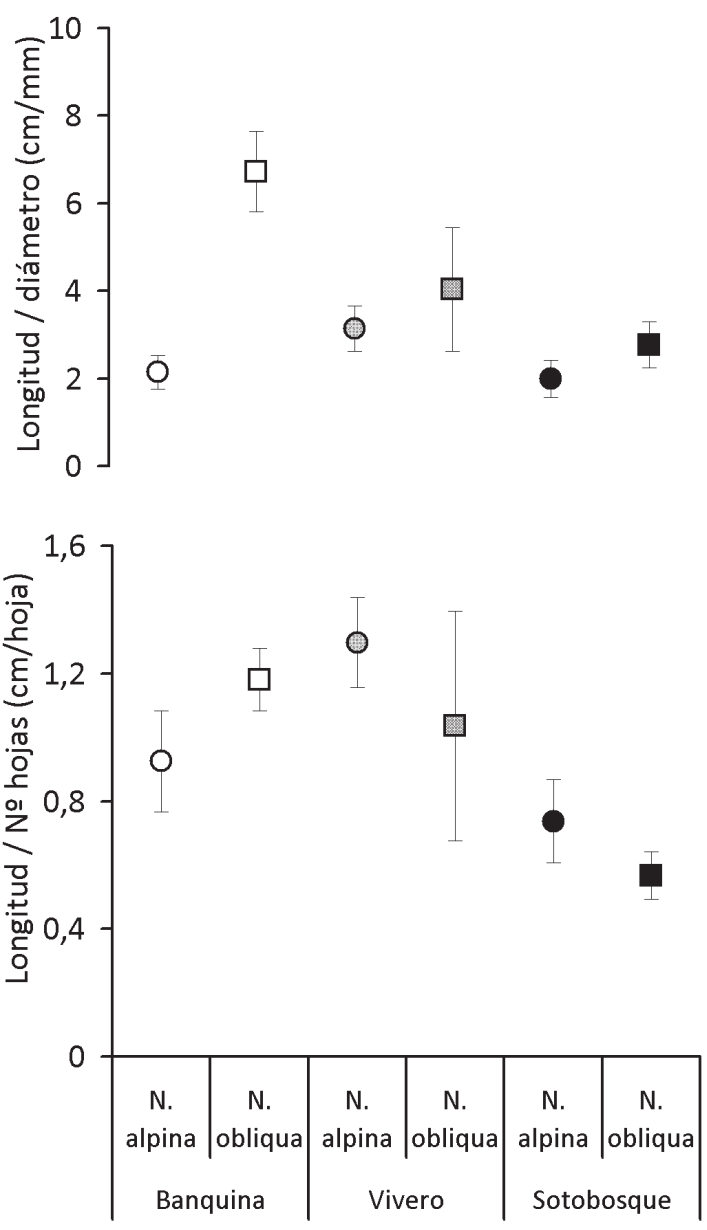

Fig. 5. A: Promedio ( \pm 1 EE) de la esbeltez del tallo (longitud/diámetro). B: Longitud media de entrenudos (longitud/número de hojas) de la unidad de crecimiento de extensión más reciente (2008-2009) de las ramas principales de árboles de $N$. alpina (círculos) y $N$. obliqua (cuadrados) desarrollados en vivero, banquina y sotobosque.

$66^{\circ}$ para N. alpina y de $62^{\circ}$ para N. obliqua (Fig. 7B). Las comparaciones estadísticas de $\beta>0$ indicaron diferencias significativas entre sitios (vivero < [banquina $=$ sotobosque]; $F=23,3, p<0,001)$, entre especies ( $N$. alpina $>N$. obliqua; $F=46,3, p<0.001$ ), $\mathrm{y}$ entre posiciones de las RSs sobre la UC portadora (mayores para RSs proximales que para RSs distales; $F=164,6, p<0,001)$. Las variaciones de $\beta$ entre las tres RSs más distales fueron notables (Fig. 7B; no se presentan los datos para RSs más proximales, pero se los puede solicitar al primer autor). No se encontraron efectos significativos
Tabla 3. Efectos del sitio de crecimiento, de la especie y de la interacción entre ambos factores (Sitio x Esp.) sobre el número ( $N^{\circ} \mathrm{RSs}$ ) y la suma de los diámetros basales $\left(\sum R S_{d}\right)$ de las ramas secundarias derivadas de UCs de las ramas principales, mediante ANCOVA de dos factores; en ambos casos se incluyeron la edad de la rama principal (Edad) y el número de hojas de la UC de la rama principal ( $N^{0}$ hojas) como covariables. También se indican los efectos de los mismos factores y del nivel de observación (Nivel Obs. UC de la rama principal extendida en 2007-2008 y rama principal completa) sobre la relación entre $\Sigma R S_{d}$ y el diámetro de la UC portadora de la rama principal ( $\Sigma R S_{d} /$ diam. RP); la edad de la rama principal fue incluida como covariable.

\begin{tabular}{|c|c|c|c|c|c|c|}
\hline \multirow[b]{2}{*}{ Factor } & \multicolumn{2}{|c|}{$\mathrm{N}^{\circ} \mathrm{RSs}$} & \multicolumn{2}{|c|}{$\Sigma \mathrm{RS}_{\mathrm{d}}$} & \multicolumn{2}{|c|}{$\Sigma \mathrm{RS}_{\mathrm{d}} /$ diam. RP } \\
\hline & $F$ & $P$ & $F$ & $P$ & $F$ & $P$ \\
\hline Sitio & 3,5 & * & 4,7 & * & 6,3 & ns \\
\hline Especie & 0,3 & $n s$ & 4,9 & * & 61,3 & ** \\
\hline Sitio x Esp. & 0,3 & $n s$ & 0,5 & ns & 0,1 & ns \\
\hline Nivel Obs. & $\mathrm{N} / \mathrm{A}$ & & $\mathrm{N} / \mathrm{A}$ & & 40,2 & *** \\
\hline \multicolumn{7}{|l|}{ Covariable } \\
\hline Edad & 0,4 & $n s$ & 0,0 & ns & 0,3 & ns \\
\hline$N^{0}$ hojas & 65,3 & $* * *$ & 72,2 & $* * *$ & $\mathrm{~N} / \mathrm{A}$ & \\
\hline
\end{tabular}

Referencias: ${ }^{* *} P<0,001,{ }^{* *} P<0,01,{ }^{*} P \leq 0,05$, ns $P>0,05$.

del año de extensión de la UC portadora $(F=0,1$, $p>0,1)$ o de la interacción sitio $\mathrm{x}$ especie $(F=1,5$, $p>0,1)$ sobre $\beta$. La frecuencia de RSs con $\beta=0$ fue similar para ambas especies $(\chi=2,3, p>0,1$; todos los sitios combinados), pero fue mayor en árboles del sotobosque que en los de banquina y de vivero $(\chi=30,1, p<0,001$; ambas especies combinadas).

\section{Discusión}

Crecimiento de las ramas principales de Nothofagus en diferentes ambientes

En la presente evaluación retrospectiva del crecimiento de las ramas principales de $N$. alpina y $N$. obliqua se pusieron en evidencia notables diferencias entre árboles desarrollados bajo condiciones lumínicas contrastantes. Desde su iniciación a partir del tronco, las ramas principales de los árboles de estas especies crecieron menos en 


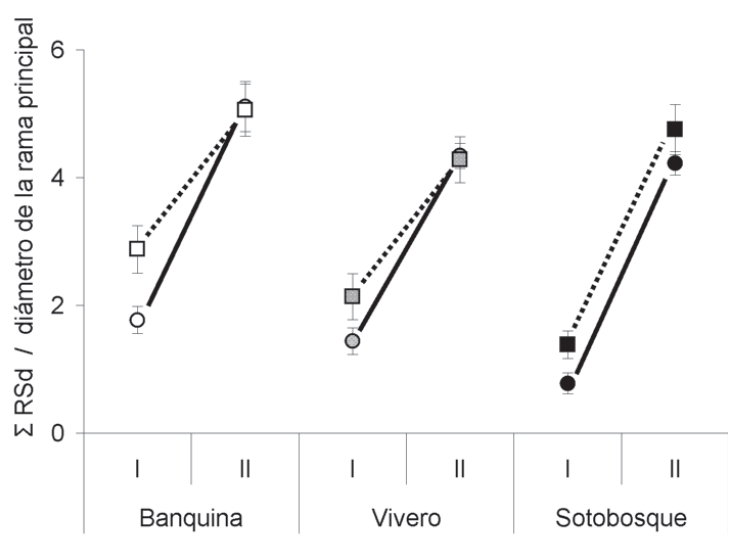

Fig. 6. Promedio ( $\pm 1 \mathrm{EE})$ de la relación entre la suma de los diámetros de las ramas secundarias y el diámetro de la rama principal portadora ( $\Sigma R S_{\mathrm{d}} /$ diám. $\mathrm{RP}$ ) calculada para (I) la unidad de crecimiento ramificada más distal de la rama principal (extendida en 2007-2008) y (II) la rama principal completa (incluyendo en este caso todas las ramas secundarias vivas derivadas de ésta) para árboles de $N$. alpina (círculos) y $N$. obliqua (cuadrados).

longitud y diámetro en condiciones de sotobosque que en los dos sitios menos sombreados (banquina y claros de bosque). En concordancia, las UCs más recientes de esas ramas fueron más delgadas y cortas y presentaron menos hojas que las de ramas principales desarrolladas en ambientes menos sombreados. Además, las ramas principales de los árboles del sotobosque presentaron ramas secundarias de menor tamaño y en menor número que aquellas de los árboles desarrollados en los otros dos ambientes. Esta última diferencia entre sitios se mantuvo aproximadamente constante para UCs de distintas edades. La menor capacidad de ramificación en las ramas principales de los árboles de sotobosque concuerda con el menor número de hojas $\mathrm{y}$, por lo tanto, de meristemas axilares observados en las UCs de estas ramas. El bajo vigor de las ramas principales en el ambiente más sombreado guarda relación con el nivel de tolerancia a la sombra, intermedio a bajo, atribuido a estas especies sobre bases ecológicas y fisiológicas (Read \& Hill, 1985; Weinberger \& Ramírez, 2001; Donoso et al., 2006a, b). Sin embargo, no se observaron aquí algunas de las respuestas que se han registrado en otros estudios en plantas intolerantes o evasoras de la sombra, como el desarrollo de

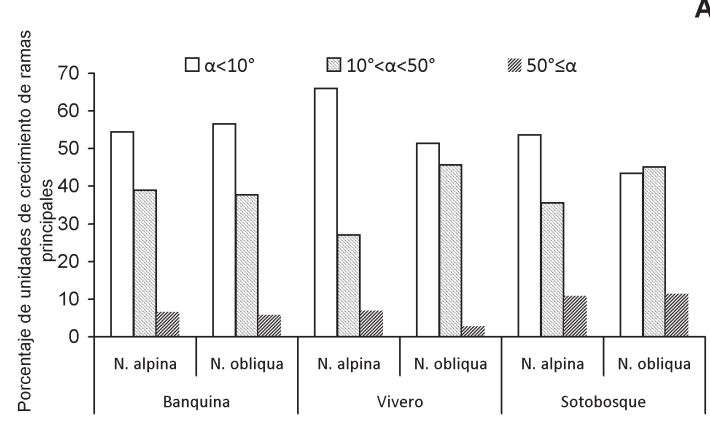

A

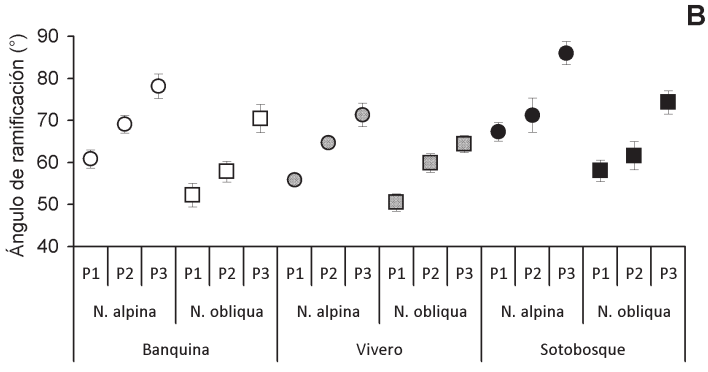

Fig. 7. A: Ángulos ( $\alpha$ ) formados entre dos unidades de crecimiento sucesivas de las ramas principales. Para cada sitio y entidad, se indican los porcentajes de unidades de crecimiento que forman $\alpha<10^{\circ}$, $10^{\circ}<\alpha<50^{\circ}$ y $50^{\circ} \leq \alpha$ con la unidad de crecimiento precedente. B: Promedio ( $\pm 1 \mathrm{EE})$ de los ángulos $(\beta)$ formados entre las ramas secundarias y las unidades de crecimiento de las ramas principales portadoras, considerando los tres nudos más distales de las unidades de crecimiento de las ramas principales ( $P 1, P 2$ and $P 3$, siendo $P 1$ el nudo más distal), discriminados por especie y sitio de crecimiento. Sólo se incluyen $\beta>0$ (véase el texto para más detalles).

entrenudos largos y de tallos más esbeltos (o sea con mayor relación longitud/diámetro; Henry \& Aarssen, 1997; Henry \& Thomas, 2002; Valladares \& Niinemets, 2008 y referencias allí incluidas; pero ver Charles-Dominique et al., 2012 para un resultado discordante). Por otro lado, merece destacarse el hecho de que el vigor de las ramas principales de ambas especies de Nothofagus no se incrementó directamente con la disponibilidad de luz. Los resultados parecen indicar que condiciones intermedias de luminosidad serían las óptimas para el desarrollo de las ramas principales de estas especies.

Es interesante mencionar el notable efecto de la edad de las ramas principales sobre el número de hojas que desarrollaron sus UCs, que contrasta 
con la falta de relación entre la edad de las ramas principales y la longitud de sus UCs (Tabla 2). Estos resultados pueden explicarse si se toma en cuenta que, en las especies de Nothofagus, el número de hojas de las UCs es función, fundamentalmente, del proceso de organogénesis que ocurre en las yemas al final de una estación de crecimiento (García et al., 2006). Si bien las condiciones ambientales durante la organogénesis podrían afectar el número de hojas preformadas (Diggle, 2002), este número tendría un fuerte componente ontogenético: la preformación estaría muy condicionada por la expresión, al momento de la organogénesis, de una fase del gradiente morfogenético la cual se relaciona, a su vez, con la edad del eje (Barthélémy \& Caraglio, 2007). La longitud de cada UC, por otro lado, depende no sólo de la organogénesis que ocurre al final de una estación de crecimiento, sino también del alargamiento de los entrenudos durante la estación de crecimiento siguiente. Nuestros resultados indican que, en estas especies, el número de hojas de un eje sería un atributo determinado principalmente por factores endógenos, mientras que la longitud de un eje estaría afectada más notablemente por factores ambientales, en concordancia con estudios anteriores sobre Nothofagus (Passo et al., 2002; Stecconi et al., 2010) y otras especies (Taugourdeau \& Sabatier, 2010).

Parte de las diferencias entre sitios en cuanto al tamaño de las ramas principales y sus UCs componentes, observadas en este estudio, podrían deberse a que los árboles muestreados expresaban diferentes fases ontogenéticas al momento de realizado el muestreo, a pesar de que, por apreciación visual, todos ellos se encontraban en la etapa de desarrollo de árbol juvenil o juveniladulto (Barthélémy et al. 1999). De acuerdo al conocimiento actual sobre el desarrollo de los árboles de Nothofagus (Stecconi et al., 2010), es probable que las ramas principales de los árboles del vivero estuvieran expresando una transición marcada entre la fase temprana, de crecimiento vigoroso, y una fase intermedia, de crecimiento moderado. En contraste, las ramas principales de los árboles del sotobosque habrían estado expresando, por más de diez años, una fase ontogenética más avanzada, de menor vigor. Los árboles de la banquina habrían estado en fases intermedias respecto de aquellas observadas en los árboles de los otros dos sitios.
A pesar del menor desarrollo de las ramas en el sotobosque, los árboles de $N$. alpina y $N$. obliqua son capaces de vivir en ese ambiente por períodos prolongados (se observaron árboles de sotobosque de más de 30 años de edad). Esto podría sugerir que, en árboles de sotobosque, la tendencia al desarrollo de ramas con UCs más pequeñas y menos ramificadas que las de árboles menos sombreados, estaría vinculada más a una estrategia de "espera" que a una de "evasión" de la sombra (como se encontró en otras especies de árboles; Kubota \& Hara, 1995, 1996). En las especies de Nothofagus la regeneración por crecimiento de plántulas es abundante luego de la formación de claros grandes en el dosel (e.g. Suárez \& Kitzberger, 2010). No obstante, la presencia en el sotobosque de ejemplares como los analizados en este estudio, notablemente menores en edad y tamaño comparados con aquellos que componen el dosel, sugiere que, al menos bajo determinadas circunstancias, la regeneración bajo el dosel es posible.

\section{Desarrollo jerárquico de la ramificación}

La arquitectura de una planta depende en buena medida de la disposición de las ramas derivadas de cada eje y del tamaño de esas ramas en relación al tamaño del eje portador. Ambos atributos de la ramificación determinan el nivel de diferenciación o de jerarquía que se establece en un sistema ramificado (Suzuki \& Suzuki, 2009). En las ramas principales de los árboles de los tres sitios considerados en este estudio, las UCs más recientemente extendidas exhibieron probabilidades de ramificación progresivamente menores hacia su extremo proximal. La diferencia en la portación de ramas entre nudos distales y nudos proximales se fue acentuando a medida que aumentó la edad de la UC portadora (Fig. 4C, D), debido a que la muerte de ramas secundarias (por autopoda) fue más frecuente entre aquellas de posición más proximal (evidenciada por la presencia de ramas muertas o de sus cicatrices sobre las ramas principales). De esta forma, con el aumento de edad de una rama principal se va acentuando la acrotonía de ramificación de sus UCs, una de las características de la ramificación de los árboles de Nothofagus (Barthélémy et al., 1999) que, en base a este estudio, no parece ser afectada por las condiciones de crecimiento.

La producción de ramas secundarias en $N$. alpina y N. obliqua y la suma de los diámetros de 


\section{J. Puntieri et al. - Patrones de ramificación en Nothofagus}

esas ramas $\left(\Sigma \mathrm{RS}_{\mathrm{d}}\right)$ fueron menores en los árboles del sotobosque que en los árboles de los sitios menos sombreados, incluso luego de incorporar el posible efecto del número de hojas de las UCs del eje portador (o sea, los posibles nudos de origen de las ramas). A pesar de estas diferencias y de los diferentes grados de mortalidad de ramas secundarias, los ambientes con diferente sombreo no afectaron el desarrollo jerárquico de los sistemas ramificados evaluado mediante la relación entre la suma de los diámetros de las ramas secundarias y el diámetro de la UC portadora de la rama principal. Por otro lado, el análisis de los ángulos formados entre las ramas secundarias y las UCs de las ramas principales $(\beta)$ indica que éstos fueron más cerrados para los árboles del vivero que para los árboles del sotobosque y de banquinas (considerando el efecto altamente significativo de la posición de las ramas sobre las UCs portadoras). Aunque los árboles del sotobosque tendieron a presentar ramas orientadas en ángulos más abiertos que los de la banquina (Fig. 7B), esta tendencia no alcanzó niveles significativos. De modo que nuestros resultados no apoyan la idea de que la estructura jerárquica de los sistemas ramificados de estas especies es afectada por la luminosidad del ambiente y, en consecuencia, se oponen a lo indicado en estudios previos sobre otras especies arbóreas (Fisher, 1986; Givnish, 1995; Luken et al., 1995; Huber \& Stuefer, 1997; Delagrange et al., 2006; Charles-Dominique et al., 2012).

La estructura del sistema de ramas de los árboles incluye un componente ontogenético que puede determinar variaciones en la estructura jerárquica de los ejes según la edad de los mismos. Este componente ontogenético fue incluido en el presente estudio sobre $N$. alpina y $N$. obliqua a través del análisis de la ramificación de las ramas principales a dos niveles de observación: se consideraron, por un lado, UCs recientemente extendidas y, por otro lado, la ramificación completa desde el inicio de las ramas principales a partir del tronco. Los resultados aquí presentados destacan la importancia de dicho componente ontogenético en la jerarquía de los sistemas ramificados de $N$. alpina y $N$. obliqua: a medida que una rama principal adiciona nuevas UCs e incrementa su diámetro por actividad cambial, aumenta el tamaño (en diámetro basal) del conjunto de ramas secundarias en relación al tamaño de la rama principal que las porta. Además, dado que el ángulo de inserción de las ramas secundarias es mayor en ramas proximales que en ramas distales, es esperable que la autopoda de las ramas proximales redunde en la disminución del ángulo promedio de inserción (organización menos jerárquica) con el aumento de la edad de la rama principal. En conclusión, las ramas principales de $N$. alpina y $N$. obliqua se vuelven sistemas menos jerárquicos al incrementarse su edad, sin importar el sitio de desarrollo del árbol (Fig. 6). Estos resultados indican que los cambios que se producen en la arquitectura de los árboles a lo largo de su ontogenia pueden vincularse con variaciones en la estructura jerárquica de los sistemas ramificados en desarrollo (Barthélémy et al., 1989; CharlesDominique et al., 2012).

Es interesante destacar que la proporción de ramas secundarias que siguieron la línea de crecimiento de la UC portadora $(\beta=0)$ fue mayor en los árboles del sotobosque que en los de los otros dos sitios del presente estudio. Esto implica que en un ambiente sombreado, es mayor la probabilidad de que una rama derivada de una rama principal siga la línea de desarrollo de esta última pero sin constituir un verdadero relevo de la UC portadora sino como un eje subordinado (Fig. 1B). En tales casos, una rama más proximal de la misma UC de la rama principal se desarrolla en mayor medida, constituyendo la continuación de la rama principal aunque en forma más sinuosa (ver Barthélémy et $a l ., 1999)$. Eventos de subordinación de una rama distal de una UC por el mayor crecimiento de una rama más proximal de la misma UC podrían relacionarse con la ocurrencia de micrositios de muy baja disponibilidad lumínica. El crecimiento de una UC de una rama principal en uno de esos micrositios significaría la pérdida de vigor de la rama más distal de la UC y el desarrollo de un relevo desde una de las ramas más proximales.

Diferencias inter-especificas en la estructura de las ramas principales

En este estudio se observaron varias diferencias entre $N$. alpina y $N$. obliqua respecto de la arquitectura de sus ramas principales y sus respuestas ante diferentes condiciones de crecimiento. Luego de alcanzar su extensión completa, las UCs de las ramas principales de ambas especies no difieren en longitud. El desarrollo de tallos más gruesos en relación a su longitud en $N$. alpina se 
relacionaría con una mayor capacidad auto-portante en comparación con la de $N$. obliqua. En esta última especie, la mayor esbeltez (= relación longitud/ diámetro) de los brotes antes del crecimiento secundario determinaría menor capacidad de crecer horizontalmente y explicaría la tendencia de sus ramas principales a curvarse, orientando el extremo distal hacia el suelo. Las interacciones significativas entre sitio de crecimiento y especie para la longitud y el número de hojas de ramas principales completas, y para el número de hojas de sus más recientes UCs, indicarían que el efecto negativo del desarrollo en el sotobosque es más notable en $N$. obliqua que en $N$. alpina, en acuerdo con la menor tolerancia a la sombra atribuida a $N$. obliqua (Veblen et al., 1981; Donoso et al., 2006a, b). Por otro lado, las tendencias a disminuciones en la longitud media de los entrenudos y la esbeltez del tallo más marcadas con mayor nivel de sombreo en la especie supuestamente menos tolerante a la sombra ( $N$. obliqua), contrastan con las predicciones de algunos estudios previos sobre la respuesta de plantas con diferente tolerancia a la sombra a condiciones de baja luminosidad (ver Henry \& Aarssen, 1997; Valladares \& Niinemets, 2008).

Las respuestas cualitativas de los sistemas de ramas ante diferentes condiciones de crecimiento fueron similares para las dos especies incluidas en este estudio. Al considerar las UCs ramificadas más recientemente extendidas, el número de ramas secundarias y la suma de sus diámetros $\left(\Sigma \mathrm{RS}_{\mathrm{d}}\right)$ fueron mayores en $N$. obliqua que en $N$. alpina. Las variaciones entre sitios en el número de ramas y en la suma de sus diámetros fueron similares para las dos especies. La suma de los diámetros de las ramas secundarias relativa al diámetro de la UC portadora fue, en promedio, mayor en $N$. obliqua que en $N$. alpina. Además, las ramas secundarias formaron ángulos más abiertos con las UCs portadoras en $N$. alpina que en $N$. obliqua. Estos resultados indican que el sistema de ramificación es más jerárquico en $N$. alpina que en $N$. obliqua, independientemente de las condiciones lumínicas del sitio de crecimiento. Esta diferencia jerárquica entre los sistemas ramificados de ambas especies concuerda con la distinción entre ellas en cuanto a la diferenciación entre el tronco y sus ramas principales, que fuera destacada por Deans et al. (1992) al ponderar el uso de $N$. alpina con fines forestales. Estudios previos sugieren que especies identificadas con etapas sucesionales más tempranas (menos tolerantes a la sombra) se ramifican más profusamente que especies de etapas más tardías (especies más tolerantes; Seiwa et al., 2006). En este sentido, los resultados alcanzados por el presente estudio apoyan las observaciones previas que asignan mayor tolerancia a la sombra a $N$. alpina que a $N$. obliqua (Donoso, 2006).

\section{Conclusiones}

La disponibilidad de luz afecta significativamente el tamaño y la capacidad de ramificación de las ramas principales en árboles de $N$. alpina y N. obliqua. En ambas especies, las ramas principales pueden permanecer por años en árboles desarrollados en sotobosque, produciendo unidades de crecimiento cortas y poco ramificadas en relación con aquellas que se forman en sitios más abiertos. Los sistemas ramificados iniciados con las ramas principales atraviesan fases con diferente grado de desarrollo jerárquico a lo largo de su ontogenia, dependiendo del crecimiento y la muerte de las ramas por autopoda. El desarrollo de estos árboles en el sotobosque modificaría el gradiente de tamaño de las ramas generadas a partir de una UC portadora respecto del gradiente que se observa en árboles de ambientes abiertos. La especie $N$. obliqua desarrolla sistemas ramificados menos jerárquicos y mayores diferencias arquitecturales entre ambientes que $N$. alpina, lo cual concuerda con la menor tolerancia a la sombra atribuida a la primera de estas especies.

\section{Agradecimientos}

Este estudio fue financiado por los proyectos PNFOR4232 (INTA) y PIP 11220080101026 (CONICET). S. Ghirardi y C. Torres recibieron becas de CONICET durante la realización de este trabajo. Los autores agradecen a L. Gallo y a M.M. Azpilicueta (INTA Bariloche) por sus asistencias en diferentes etapas de este estudio, a S. Sabatier y D. Barthélémy por sus comentarios sobre versiones anteriores del manuscrito y a D. Barthélémy por su apoyo en el estudio de la arquitectura de Nothofagus. 


\section{J. Puntieri et al. - Patrones de ramificación en Nothofagus}

\section{Biblografía}

BARTHÉLÉMY, D., C. EDELIN, \& F. HALLÉ. 1989. Some architectural aspects of tree aging. Ann. Sci. For. 46: 194-198.

BARTHÉLÉMY, D. \& Y. CARAGLIO. 2007. Plant architecture: a dynamic, multilevel and comprehensive approach to plant form, structure and ontogeny. Ann. Bot. 99: 375-407.

BARTHÉLÉMY, D., S. SABATIER \& O. PASCAL. 1997. Le développement architectural du noyer noir, Juglans nigra L. (Juglandaceae). Forêt-entreprise 115: 40-47.

BARTHÉLÉMY, D., J. G. PUNTIERI, C. BRION, E. RAFFAELE, J. MARINO \& P. MARTINEZ. 1999. Morfología de las unidades estructurales y modo de desarrollo básico de especies patagónicas de Nothofagus (Fagaceae). Bol. Soc. Argent. Bot. 34: 29-38.

CALABRIA, C. \& J. G. PUNTIERI. 2008. Desarrollo foliar y caulinar de las unidades de alargamiento de Nothofagus dombeyi (Nothofagaceae) en condiciones de alta y baja luminosidad. Bol. Soc. Argent. Bot. 43: 19-30.

CANHAM, C. 1988. Growth and canopy architecture of shade-tolerant trees: response to canopy gaps. Ecology 69: 786-795.

CAO, K.-F. 2001. Morphology and growth of deciduous and evergreen broad-leaved saplings under different light conditions in a Chinese beech forest with dense bamboo undergrowth. Ecol. Res. 16: 509-517

CHARLES-DOMINIQUE, T., C. EDELIN \& A. BOUCHARD. 2010. Architectural strategies of Cornus sericea, a native but invasive shrub of Southern Quebec, Canada, under an open or a closed canopy. Ann. Bot. 105: 205-220.

CHARLES-DOMINIQUE, T., C. EDELIN, C. BRISSON \& A. BOUCHARD. 2012. Architectural strategies of Rhamnus cathartica (Rhamnaceae) in relation to canopy openness. Botany 90: 976-989.

CHAUBERT-PEREIRA, F., Y. CARAGLIO, C. LAVERGNE \& Y. GUÉDON. 2009. Identifying ontogenetic, environmental and individual components of forest tree growth. Ann. Bot. 104: 883-896.

CONTI, H. A. 1998. Características climáticas de la Patagonia. In: Correa, M. N. (ed.), Flora Patagónica. Vol. VIII(I), pp. 31-47. INTA, Buenos Aires.

COSTE, S., J. C. ROGGY, L. GARRAUD, P. HEURET, E. NICOLINI \& E. DREYER. 2009. Does ontogeny modulate irradiance-elicited plasticity of leaf traits in saplings of rain-forest tree species? A test with Dicorynia guianensis and Tachigali melinonii (Fabaceae, Caesalpinioideae). Ann. For. Sci. 66: doi:10.1051/forest/2009062.
DAY, J. S. 1998. Light conditions and the evolution of heteroblasty (and the divaricate form) in New Zealand. New Zeal. J. Bot. 22: 43-54.

DEANS, J. D., H. L. BILLINGTON \& F. J. HARVEY 1992. Winter frost hardiness of two Chilean provenances of Nothofagus procera in Scotland. Forestry 65: 205-212.

DELAGRANGE, S., P. MONTPIED, E. DREYER, C. MESSIER \& H. SINOQUET. 2006. Does shade improve light interception eficiency? A comparison among seedlings from shade-tolerant and shadeintolerant temperate deciduous tree species. New Phytol. 172: 293-304.

DIGGLE, P. K. 2002. A developmental morphologist's perspective on plasticity. Evol. Ecol. 16: 267-283.

DONOSO, P., C. DONOSO, P. MARCHELLI, L. GALLO \& B. ESCOBAR. 2006a. Nothofagus nervosa (Phil.) Dim. et Mil., otros nombres científicos usados: Nothofagus alpina, Nothofagus procera, Raulí, Familia: Fagaceae. In: Donoso, C. (ed.), Las especies arbóreas de los bosques templados de Chile y Argentina, pp. 448-461. Marisa Cuneo Ediciones, Valdivia.

DONOSO, P., C. DONOSO, L. GALlO, M. M. AZPILICUETA, A. BALDINI \& B. ESCOBAR. 2006b. Nothofagus obliqua (Mirb.) Oerst. Roble, Pellín, Hualle, Familia: Fagaceae. In: Donoso, C. (ed.), Las especies arbóreas de los bosques templados de Chile y Argentina, pp. 471-485. Marisa Cuneo Ediciones, Valdivia.

FISHER, J. B. 1986. Branching patterns and angles in trees. In: Givnish, T. J. (ed.), On the economy of plant form and function, pp. 493-523. Cambridge University Press, Cambridge.

FRANKLIN, K. A \& G. C. WHITELAM. 2005. Phytochromes and shade-avoidance in plants. Ann. Bot. 96: 169-175.

GARCÍA, S., J. G. PUNTIERI \& G. VOBIS. 2006. Morfología y anatomía del ápice caulinar de (Nothofagus dombeyi) a lo largo de un año. Bol. Soc. Argent. Bot. 41: 13-21.

GIVNISH, T. J. 1988. Adaptation to sun and shade: a whole plant perspective. Austr. J. Plant Physiol. 15: 63-92.

GIVNISH, T. J. 1995. Plant stems: biomechanical adaptation of energy capture and influence on species distributions. In: Gartner, B. (ed.), Plant Stems: physiology and functional ecology, pp. 3-49. Academic Press, San Diego.

GUÉDON, Y., Y. CARAGLIO, P. HEURET, E. LEBARBIER \& C. MERIDIEU. 2007. Analyzing growth components in trees. J. Theor. Biol. 248: 418-447.

GUÉRARD, N., D. BARTHÉLÉMY, A. CABANETTES, C. F. COURDIER, C. P. TRICHET \& J, WILLM. 
2001. Influence de la compétition herbacée sur la croissance et l'architecture de jeunes Chênes rouges d'Amérique (Quercus rubra L.) en plantation. Ann. For. Sci. 58: 395-410.

HALLÉ, F., R. A. A. OLDEMAN \& P. B. TOMLINSON. 1978. Tropical trees and forests. An architectural analysis. Springer-Verlag, Berlin. 441 pp.

HENRY, H. A. L. \& L. W. AARSSEN. 1997. On the relationship between shade tolerance and shade avoidance in woodland plants. Oikos 80: 575-582.

HENRY, H. A. L. \& S. C. THOMAS. 2002. Interactive effects of lateral shade and wind on stem allometry, biomass allocation, and mechanical stability in Abutilon theophrasti (Malvaceae). Amer. J. Bot. 89: 1609-1615.

HEURET, P., C. MEREDIEU, T. COUDURIER, F. COURDIER \& D. BARTHÉLÉMY. 2006. Ontogenetic trends in the morphological features of main stem annual shoots of Pinus pinaster (Pinaceae). Amer. J. Bot. 93: 1577-1587.

HORN, H. S. 1971. The adaptive geometry of trees. Princeton University Press, Princeton. 144 pp.

HUBER, H. \& J. F. STUEFER. 1997. Shade-induced changes in the branching pattern of a stoloniferous herb: functional response or allometric effect? Oecol. 110: 478-486.

ISHIHARA, M. \& K. KIKUZAWA. 2009. Annual and spatial variation in shoot demography associated with masting in Betula grossa: comparison between mature trees and saplings. Ann. Bot. 104: 1195-1205.

KUBOTA, Y. \& T. HARA. 1995. Tree competition and species coexistence in a sub-boreal forest, Northern Japan. Ann. Bot. 76: 503-512.

KUBOTA, Y. \& T. HARA. 1996. Allometry and competition between saplings of Picea jezoensis and Abies sachalinensis in sub-boreal coniferous forest, northern Japan. Ann. Bot. 77: 529-537.

KÜPPERS, M. 1994. Canopy gaps: competitive light interception and economy space filling - a matter of whole-plant allocation. In: Caldwell, M. M. \& R. W. Pearcy (eds.), Exploitation of environmental heterogeneity by plants, pp. 111-144. Academic Press, London.

LUKEN, J. O., T. C. THOLEMEIER, B. A. KUNKEL \& L. M. KUDDES. 1995. Branch architecture plasticity of Amur honeysuckle (Lonicera maackii (Rupr.) Herder): Inicial response in extreme light environments. Bull. Torrey Bot. Club 122: 190-195.

NIKLAS, K. J. 2007. Maximum plant height and the biophysical factors that limit it. Tree Physiol. 27: 433-440.

OLDEMAN, R. A. A. 1989. Biological implications of leguminous tree architecture. Monogr. Syst. Bot. Missouri Bot. Gard. 29: 17-34.

PASSO, A., J. G. PUNTIERI \& D. BARTHÉLÉMY. 2002.
Trunk and main-branch development in Nothofagus pumilio (Nothofagaceae): a retrospective analysis of tree growth. Canad. J. Bot. 80: 763-772

PICKETT, S. T. A. \& J. S. KEMPF. 1980. Branching patterns in forest shrubs and understory trees in relation to habitat. New Pytol. 86: 219-228.

PUNTIERI, J. G. \& S. GHIRARDI. 2010. Growth-unit structure in trees: effects of branch category and position in Nothofagus nervosa, $N$. obliqua and their hybrids (Nothofagaceae). Trees 24: 657-665.

PUNTIERI, J. G., M. S. SOUZA, D. BARTHÉLÉMY, C. MAZZINI \& C. BRION. 2003. Axis differentiation in two South American Nothofagus species (Nothofagaceae). Ann. Bot. 92: 589-599.

PUNTIERI, J. G., J. GROSFELD, M. STECCONI, M. M. AZPILICUETA, L. GALLO, C. BRION \& D. BARTHÉLÉMY. 2007. Shoot development and dieback in progenies of Nothofagus obliqua. Ann. For. Sci. 64: 839-844.

READ, J. \& R. S. HILL. 1985. Photosynthetic responses to light of Australian and Chilean species of Nothofagus and their relevance to the rainforest dynamics. New Phytol. 101: 731-742.

SABATIER, S. \& D. BARTHÉLÉMY. 1999. Growth dynamics and morphology of annual shoots, according to their architectural position, in young Cedrus atlantica (Endl.) Manetti ex Carrière (Pinaceae). Ann. Bot. 84: 387-392.

SCHMITT, J., J. R. STINCHCOMBE, M. S. HESCHEL \& H. HUBER. 2003. The adaptive evolution of plasticity: phytochrome-mediated shade avoidance responses. Integrative Compar. Biol. 43: 459-469.

SCOPPA, C. O. 1998. Los suelos. In: M.N. Correa (ed.), Flora Patagónica. pp. 15-30. Colección Científica del INTA, Buenos Aires.

SEIWA, K., K. KIKUZAWA, T. KADOWAKI, S. AKASAKA \& N. UENO. 2006. Shoot life span in relation to successional status in deciduous broadleaved tree species in a temperate forest. New Phytol. 169: 537-548.

STECCONI, M., J. G. PUNTIERI \& D. BARTHÉLÉMY. 2010. An architectural approach to the growth forms of Nothofagus pumilio (Nothofagaceae) along an altitudinal gradient. Botany 88: 699-709.

SUÁREZ, M. L. \& T. KITZBERGER. 2010. Differential effects of climate variability on forest dynamics along a precipitation gradient in northern Patagonia. J. Ecol. 98: 1023-1034.

STEINGRAEBER, D. A. 1982. Phenotypic plasticity of branching pattern in sugar maple (Acer saccharum). Amer. J. Bot. 69: 638-648.

STEINGRAEBER, D. A., L. J. KASCHT \& D. H. FRANCK. 1979. Variation of shoot morphology and bifurcation ratio in sugar maple (Acer saccharum) saplings. Amer. J. Bot. 66: 441-445. 


\section{J. Puntieri et al. - Patrones de ramificación en Nothofagus}

STEVENS, S. G. \& A. L. PERKINS. 1992. The branching habits and life history of woody plants. Amer Natur. 139: $267-275$

SUZUKI, A. A. 2003. Shoot growth patterns in saplings of Cleyera japonica in relation to light and architectural position. Tree Physiol. 23: 67-71.

SUZUKI, A. A. \& M. SUZUKI. 2009. Why do lower order branches show greater shoot growth than higher order branches? Considering space availability as a factor affecting shoot growth. Trees 23: 69-77.

TAUGOURDEAU, O. \& S. SABATIER. 2010. Limited plasticity of shoot preformation in response to light by understorey saplings of common walnut (Juglans regia). AoB Plants doi:10.1093/aobpla/plq022.

TOMLINSON, P. B. 1987. Architecture of tropical plants. Ann. Rev. Ecol. Syst. 18: 1-21.

TORRES, C., J. G. PUNTIERI \& M. STECCONI. 2009. Estudio comparativo del crecimiento y la ramificación de brotes anuales en dos especies de Nothofagus (Nothofagaceae) y en híbridos interespecíficos. Bol. Soc. Argent. Bot. 44: 287-303.

UMEKI, K. \& T. SEINO. 2003. Growth of first-order branches in Betula platyphylla saplings as related to the age, position, size, angle, and light availability of branches. Canad. J. For. Res. 33: 1276-1286

VALLADARES, F. \& Ü. NIINEMETS. 2007. The architecture of plant crowns: from design rules to light capture and performance. In: Pugnaire, F. \& F. Valladares (eds.), Functional Plant Ecology, pp. 101-149. Taylor and Francis, New York.

VALLADARES, F. \& Ü. NIINEMETS. 2008. Shade tolerance, a key plant feature of complex nature and consequences. Ann. Rev. Ecol. Evol. Syst. 39: 237-57.
VALLADARES, F., A. SALDAÑA \& E. GIANOLI. 2011. Costs versus risks: Architectural changes with changing light quantity and quality in saplings of temperate rainforest trees of different shade tolerance. Austral Ecol. doi:10.1111/j.14429993.2011.02245.x

VEBLEN, T. T., C. DONOSO, F. M. SCHLEGEL \& B. ESCOBAR. 1981. Forest dynamics in South-Central Chile. J. Biogeogr. 8: 211-247.

VEBLEN, T. T., C. DONOSO, T. KITZBERGER \& A. J. REBERTUS. 1996. Ecology of southern Chilean and Argentinean Nothofagus forests. In: Veblen, T. T., R. S Hill. \& J. Read (eds.), The ecology and biogeography of Nothofagus forests, pp. 293-353. Yale University Press, Yale

VENABLES, W. N., D. M. SMITH \& THE DEVELOPMENT CORE TEAM. 2010. An Introduction to $R$. http://www.r-project.org/.

WEINBERGER, P. \& C. RAMÍREZ. 2001. Microclima y regeneración natural de raulí, roble y coigüe (Nothofagus alpina, N. obliqua y $N$. dombeyi). Bosque 22: 11-26

ZAR, J. R. 1999. Biostatistical analysis. New Jersey, Prentice Hall.

Recibido el 23 de julio de 2012, aceptado el 1 de noviembre de 2012. 
